\title{
Lo Hedónico y la Exhibición Ostentosa en Festivales de Música Pagos
}

\author{
Diego Alejandro Velandia-Granados ${ }^{1}$ y Myriam Carmenza \\ Sierra-Puentes ${ }^{1}$ \\ ${ }^{1}$ Fundación Universitaria Konrad Lorenz, Colombia \\ diegoa.velandiag@konradlorenz.edu.co; myriamc.sierrap@konradlorenz.edu.co| \\ https://orcid.org/0000-0003-1766-4551; https://orcid.org/0000-0002-9829-2827
}

\begin{abstract}
Resumen: El objetivo central de este estudio es: Identificar las prácticas exhibitorias ostentosas y hedónicas en un festival de música pago en Bogotá Colombia. A través de una investigación cualitativa, con triangulación múltiple, se recopiló información con 12 entrevistas, una triada a asistentes, nueve observaciones, 20 artículos web y 81 fotografías. Para el análisis se empleó teoría fundamentada que da a conocer las emociones que emergen de amistad, felicidad y euforia, aspectos de disfrute, deseos y sueños cumplidos inducidos esencialmente por artistas, componentes hedónicos que contribuyen a la experiencia y estimulación sensorial (espacios, luces, sonido, entre otros); además de la elección de la ropa como principal mecanismo para suscitar notoriedad, prestigio y aprobación, en contexto al evento como un encuentro social.
\end{abstract}

Palabras clave: Psicología del Consumidor; Festivales de Música; Consumo Hedónico; Consumo Conspicuo.

\section{The Hedonic and the Ostental Exhibition in Paid Music Festivals}

\begin{abstract}
The main objective of this study is: To identify the ostentatious and hedonic exhibitionist practices in a paid music festival in Bogotá Colombia. Through a qualitative research, with multiple triangulation, information was collected with 12 interviews, a triad of attendees, nine observations, 20 web articles and 81 photographs. Grounded theory was used for the analysis, which reveals the emotions that emerge from friendship, happiness and euphoria, aspects of enjoyment, desires and dreams fulfilled essentially induced by artists, hedonic components that contribute to the experience and sensory stimulation (spaces, lights, sound, among others); in addition to the choice of clothing as the main mechanism to arouse notoriety, prestige and approval, in the context of the event as a social gathering.
\end{abstract}

Keywords: Consumer Psychology; Music Festival; Hedonic Consumption; Conspicuous Consumption.

\section{Introducción}

Tradicionalmente se ha considerado que el acto de compra se encuentra vinculado a satisfacer una necesidad primaria, sin embargo, a partir del estudio del consumo se identifican aspectos que influyen e incluso se convierten en la principal razón en la elección de bienes y servicios (Neme y Rodríguez-González, 2013), por ejemplo lo relacionado a lo hedónico, vinculado a experiencias emocionales (Kronrod, Gristein, \& Wathieu, 2011) así como las prácticas ostentosas donde se consumen bienes observables para señalar la riqueza a los demás (Perez-Truglia, 2013).

Cabe señalar que las principales investigaciones de consumo hedónico se han centrado en la categoría de comida, por otro lado, las prácticas ostentosas están inmersas en lo conspicuo del cual el estatus, lujo y moda son los temas más abordados. Es de ahí, que el estudio de estos constructos toma relevancia, debido a que se presentan otros escenarios y que a hoy no se han explorado, como es el caso de los festivales de música que son "eventos gracias a los cuales la cultura se revitaliza, reinventándose a sí misma por medio de un despliegue lúdico de sus imaginarios culturales" (Instituto de Políticas de Desarrollo, 2013 p.5) en especial de aquellos festivales pagos que "presentan una dependencia muy fuerte con relación al aporte de sponsors: son casi una condición de posibilidad para su realización y pueden atentar contra la sostenibilidad y construcción de marca de un festival" (Cultura Argentina, 2017 p.7). 
En esa línea, se ha evidenciado interés en el desarrollo de festivales pagos, como es el caso del festival bogotano, el cual abrió sus puertas en el año 2010 contando con solo 3.000 asistentes generando problemas económicos (Semana, 2014). A pesar de ello, la última edición, contó con 90.000 asistentes durante tres días (Tikitakas, 2019) cifras considerables, siendo el único evento nacional equiparable a otros del mundo. Respecto a las investigaciones desarrolladas en esta categoría, resulta lógico que se centran en países como Estados Unidos, Australia y el Reino Unido, debido a que es desde allí, donde parten los más importantes eventos de este tipo.

Debido a la ausencia de investigaciones en Latinoamérica de festivales de música y la importancia de vislumbrar al consumidor; se propone esta investigación para dar respuesta a la pregunta ¿cuáles son las prácticas exhibitorias ostentosas y hedónicas de los asistentes de un festival colombiano como representación de los festivales de música pagos? Con el fin de comprender al usuario de esta categoría, aportando al estudio de estos constructos teóricos en el campo de la psicología del consumidor, así como contribuciones para estrategias y tácticas de marketing.

En su estructura, los festivales pasaron de ser un evento festivo dirigido a una población local a transformarse en un espacio de oferta de servicio musical y cultural, obteniendo relevancia para empresarios, así como, para población con poder adquisitivo y estatus social por la dinámica en las relaciones sociales que emergen a su alrededor (Tabuenca, 2002). Así mismo, se consideran espacios en que los asistentes como lo mencionan Kim \& Jamal (2007) "están libres de las restricciones de la vida diaria y pueden comportarse de una manera no regida por las normas sociales convencionales que estructuran la vida cotidiana" (p. 184). Es por ello que, en estos eventos, los participantes buscan vivir experiencias que les permita encontrar sentido a lo personal y a diversos roles sociales (Kim \& Jamal, 2007).

Un estudio desarrollado por Skandalis, Byrom y Banister (2019) demostró que los asistentes a un festival de música perciben una experiencia extraordinaria, a pesar que se desarrolla dentro de tensiones estructurales y antiestructurales del mercado, como el comercio y la comunicación, siendo un tema relevante para el marketing experiencial. Sin embargo, aunque los individuos expresan sus preferencias por bienes, servicios y experiencias, estas no son perfiladas aisladamente por cada sujeto; son, más bien, la expresión que emerge de grupos sociales que irrumpen de un determinado espacio cultural y económico (García-Ruiz, 2010).

De ahí que la búsqueda de experiencias (Skandalis, et. al. 2019 y la importancia en la población con cierto poder adquisitivo (Tabuenca, 2002) hacen de los festivales de música pagos un aspecto de gran interés, siendo pertinente estudiar al asistente que está influenciado por respuestas hedónicas y a su vez lograr identificar las prácticas ostentosas que allí emergen.

Es importante indicar que el acto de compra se consideraba como un proceso económicamente racional, sin embargo, a través del estudio del consumidor, estos supuestos permitieron ampliar el campo a través de investigaciones científicas. Es así como a partir de otro enfoque, se habla del consumo hedónico, entendido como aquel centrado en la teoría de las tres "f's" que representan fantasies (fantasías), relacionada a la imaginación, deseos y sueños; feeling (sentimientos) reflejados en las emociones como la alegría, amor, miedo o dolor; y por último fun (diversión) generado por el placer producto del disfrute estético o de las actividades lúdicas (Holbrook, 2006).

De otra parte, se contrapone el consumo utilitario, que está motivado a satisfacer la necesidad a través de su función primaria, y generalmente se identifican como productos o servicios necesarios (Choi, Li, Rangan, Chatterjee \& Singh, 2014). Es de aclarar que algunas situaciones de compra son más predispuestas a provocar resultados hedónicos que otras, puesto que, es probable que un servicio de mensajería, que está orientado a resultado, conlleven a efectos utilitarios, a diferencia de adquisición de servicios recreativos que concebirá mayor expectativa a resultados hedónicos (Laplante \& Downie, 2011). 
En lo que respecta a la exhibición ostentosa, Thorstein Veblen plantea el consumo conspicuo desde su libro La teoría de la clase ociosa (1899), entendido como la manifestación de poder adquisitivo por medio de la compra de productos que son visibles, es decir que tiene como fin exhibir obligatoriamente la capacidad económica que se tiene ante la sociedad (Neme y Rodríguez-Gonzáles, 2013).

Por consiguiente, la exhibición ostentosa se convierte en una práctica para aumentar la reputación y aceptación social, recurriendo a diversos métodos como por ejemplo las fiestas y espacios de diversión (Veblen, 2000). Es de notar que este tipo de prácticas se manifiesta esencialmente en aquellos lugares donde hay mayor movilidad de población y es más extenso el contacto humano (Veblen, 2000), ello se debe a que en la actualidad y en especial, en las sociedades democráticas, estas prácticas están enlazadas por dos aspectos en los que se trabaja constantemente: la importancia social y los logros personales (Botton, 2004).

Es normal que las personas deseen lo que tienen otras, sin embargo, estas comparaciones se dan principalmente con iguales (es decir, con individuos que tienen características sociales similares a las propias) pues siempre están en una constante lucha por sobresalir y alcanzar las mismas condiciones para llegar incluso a superarlas. No obstante, cuando se elevan estas expectativas se incrementa la probabilidad al fracaso, lo que conlleva a su vez al riesgo de no ser aceptados por la sociedad y alcanzar el amor social (Botton, 2004).

Por otra parte, al exhibir consumos ostentosos no sólo se agudiza y se mantiene despierto el sentimiento de inclusión, sino que también afirma y preserva las razones para sentirse satisfecho de sí mismo; dicho de otra manera, este consumo no se alimenta únicamente del deseo de ser admirado y ser reconocido por el otro, sino que en ella surge a su vez el deseo de deleitarse de sí mismo y de lograr lo que se puede llamar como amor propio (Lipovetsky \& Roux, 2014).

Por tanto, que identificar tanto los aspectos hedónicos desde la fantasía, diversión y sentimientos como de las prácticas exhibitorias ostentosas orientadas a la visibilidad, reputación y aceptación social, toma relevancia en categorías de consumo como los festivales de música pagos, al ser estos considerados como lugares, en donde no solo se consume un tipo concreto de servicio, sino que se crea un espacio en el que engloba relaciones sociales, culturales y humanas (Prat-Forga, 2014).

\section{Metodología}

El presente estudio se desarrolló desde un enfoque cualitativo de tipo descriptivo, que permitió evaluar el desarrollo natural de los sucesos, es decir, no presentó manipulación de la realidad (Corbetta, 2003). Para incrementar la credibilidad de la investigación, se realizó triangulación de métodos y técnicas; es decir, triangulación múltiple, mostrando que fenómenos vistos desde distintos métodos ofrece mayor validez que las medidas resultantes con uno solo (Arias-Alpizar, 2011).

En la Tabla 1 se presentan las diferentes técnicas de recolección empleadas.

Tabla 1. Técnicas, fuentes e instrumentos

\begin{tabular}{|c|c|c|}
\hline & Técnica-fuente & Instrumento \\
\hline $\begin{array}{l}\text { Observación no } \\
\text { participante- } \\
\text { primaria }\end{array}$ & $\begin{array}{l}\text { Permite registrar la existencia o no de elementos } \\
\text { y aspectos considerados en los parámetros de la } \\
\text { investigación del festival (Quintana y } \\
\text { Montgomery, 2006) }\end{array}$ & Guía de observación \\
\hline $\begin{array}{l}\text { Entrevista } \\
\text { semiestructurad } \\
\text { a a profundidad- } \\
\text { primaria }\end{array}$ & $\begin{array}{l}\text { Esta técnica se enfoca en percibir las } \\
\text { perspectivas de los asistentes propios de sus } \\
\text { experiencias que son manifestadas con sus } \\
\text { mismas palabras (Taylor y Bogdan, 2000). }\end{array}$ & $\begin{array}{l}\text { Guía de entrevista a } \\
\text { asistentes, triada de } \\
\text { asistentes y asistente- } \\
\text { acompañante }\end{array}$ \\
\hline \multicolumn{3}{|c|}{ - 137 - } \\
\hline (c)) BY-NC-ND & & \\
\hline
\end{tabular}


Revisión

documental-

fuentes

secundarias
Las fuentes secundarias proporcionan una alternativa eficiente en la recopilación de datos de nuevos grupos o los mismos sujetos y se precisa como la reutilización de datos existentes para dar respuesta una pregunta de investigación (Heaton, 2004).
Páginas web relacionadas al festival del 2013 al 2019.

Fotografías de Instagram publicadas a través de hashtag del 2013 al 2019.

Fuente: Elaboración propia.

Para esta investigación se utilizó un muestreo sujeto tipo por bola de nieve con la selección de protagonistas para las fuentes primarias; además de revisión documental escrita y visual de las diferentes ediciones de un festival efectuadas entre el 2013 al 2019. El muestreo cualitativo corresponde al teórico, en el que la recolección de datos tuvo como propósito acudir a los diferentes espacios, personas y acontecimientos para generar mayor densidad en las categorías (Strauss y Corbin, 2002).

\subsection{Procedimiento}

El procedimiento se efectuó en tres momentos: El primero corresponde al diseño de instrumentos con las siguientes actividades: Diseño de guías, validación de instrumento mediante jueces, diseño de consentimiento informado, ajustes a partir de recomendaciones.

El momento 2: Ejecución de observaciones, búsqueda de participantes, consentimiento informado, aplicación de entrevistas, descarga y archivo de audios, revisión documental en páginas web y fotografías. En la Figura 1 se presenta el mapeo con las características de los participantes y las fuentes de recolección de datos.

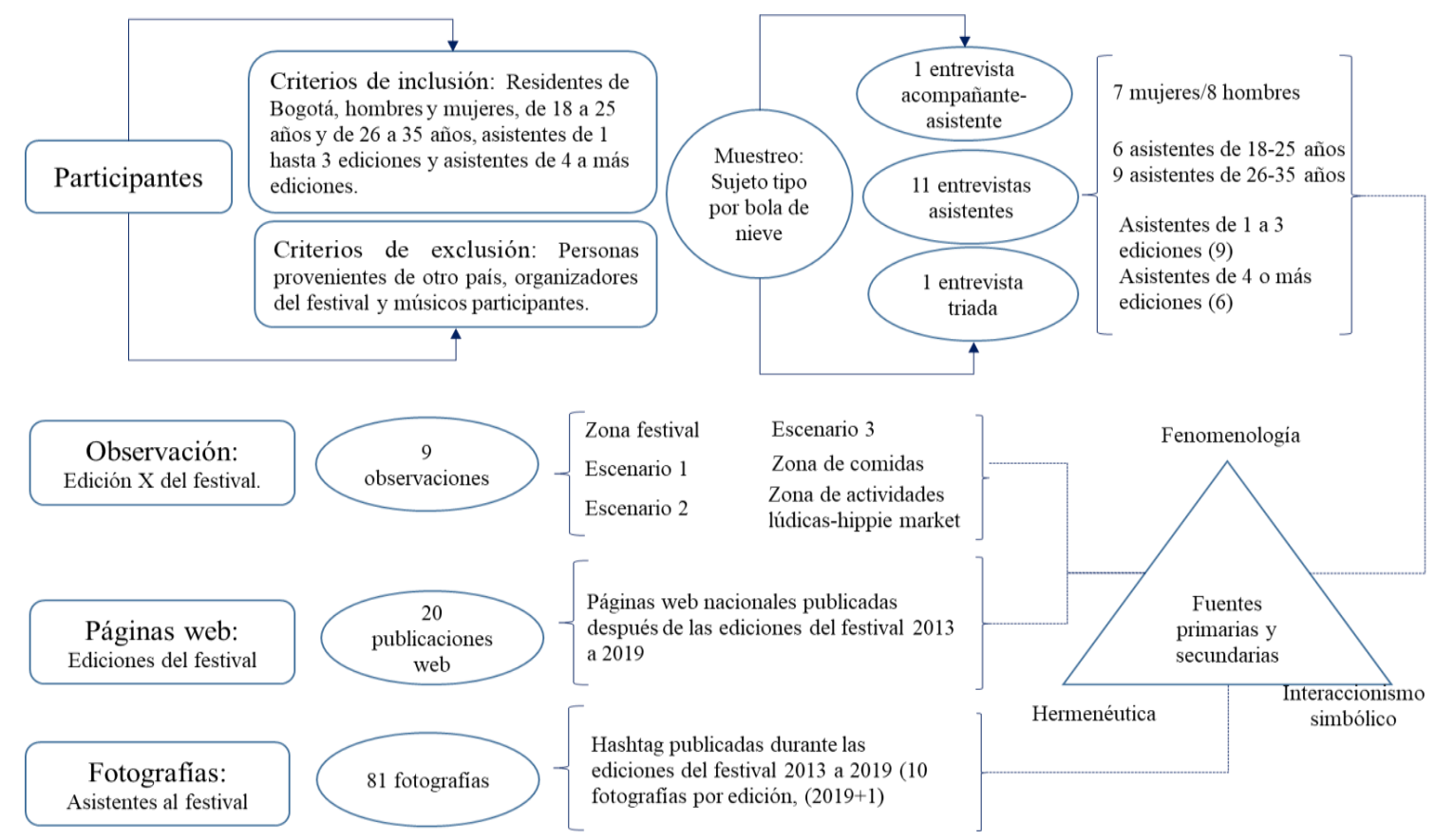

Fig. 1. Mapeo. Elaboración propia. 
La triangulación comprendió del uso de técnicas como la entrevista, triada y observación, así como de revisión documental de fuentes secundarias a partir de textos en páginas web y fotografías de redes sociales; estas se enfocaron desde los métodos fenomenológico, hermenéutico e interaccionismo simbólico, debido a que el primero es abordado desde el postulado de Husserl, en el que se describe la experiencia vivida percibida empíricamente tal y como se despliega en la conciencia, sin necesidad de recurrir a otras disciplinas o teorías (Bautista, 2011). El segundo al ser una actividad interpretativa consintió en captar el sentido de la realidad humana en diferentes contextos a través de actos, textos y comportamientos (Arráez, Calles y Moreno de Tovar, 2006) y el tercero ayudó a entender al ser humano a partir de la orientación de sus actos hacia las cosas, en función de sus significados, las cuales emergen como consecuencia de la interacción social (Walker, 2016).

Y por último el tercer momento corresponde al análisis de datos: Transcripción, codificación abierta, axial y selectiva, diseño de gráficas, análisis de datos y discusión. Los momentos dos y tres, fueron desarrollados por el investigador principal.

\subsection{Análisis de datos}

Para garantizar la baja dependibilidad se desarrolló el análisis a través de la teoría fundamentada, que busca generar conceptos y teorías a partir de datos analizados, siguiendo rigurosos procesos de comparación constante (De la Espriella \& GómezRestrepo, 2020). Este método se generó a través del software NVivo12, el cual consintió en integrar, organizar y codificar la información; con la generación de códigos abiertos que corresponde a códigos descriptivos, construyendo las primeras categorías teóricas, seguido de códigos axiales relacionando las categorías asociadas a los ejes de indagación y por último los códigos selectivos que se integraron a una categoría orientadora o categoría teórica central (Del Moral y Suárez-Relinque, 2018).

\section{Resultados}

Atendiendo a los resultados, se encuentra que el festival toma relevancia al ser considerado un espacio diseñado no solo para disfrutar de la música, sino para el desarrollo de actividades de marcas a causa de la notoriedad y posicionamiento que se pueden llegar a obtener; así como de las diversas interacciones sociales en una población principalmente joven de clase media y alta.

En lo que respecta al consumo hedónico, es concerniente a los postulados de sentimientos, diversión y fantasías (Hirschman y Holbrook, 1982), en donde florece la amistad, alegría, euforia y ansiedad por conocer lo que ofrecerá cada edición, pero también, aburrimiento y tristeza al no poder disfrutar del festival. En esa misma dirección, la diversión es adjudicada fundamentalmente a las presentaciones en vivo, pero a la vez por otras actividades de marcas y atracciones mecánicas que hacen parte de una zona diseñada como parque de diversiones. Es así como durante tres días los asistentes cantan, gritan, saltan y bailan, como declaración de diversión y disfrute concebida por el festival.

En torno a las fantasías se pueden caracterizar cuatro grupos: las relacionadas a artistas con experiencias vividas, deseos asociados a artistas, otros aspectos vividos y otros deseos. El primero integra los sueños cumplidos, al presenciar el show de los artistas preferidos, sus mensajes, la cercanía con el público y la posibilidad de traer artistas poco comerciales. El segundo se asocia a los deseos de ver a su artista favorito, la posibilidad de mejorar el cartel, así como la oportunidad de fotografiarse con artistas. En tercer lugar, se halla el impacto por los fuegos artificiales y los sectores exclusivos de las marcas, concibiendo el festival como un evento vinculado a sorpresas y cosas imprevistas. El último, indica la esperanza de contar con más géneros musicales y el anhelo de presenciar nuevas ediciones. 
De otro lado, las prácticas exhibitorias ostentosas se describen a partir de los resultados de visibilidad, reputación y aceptación social. Respecto al primero se detalla que el festival es un espacio asociado al uso de ropa de moda y verse cool, pero a la vez permite innovar en la forma de vestir, generar tendencias y ver cómo visten los demás. Para lograr resaltar en el festival, se presentan diversas alternativas como el uso de disfraces, prendas coloridas, así como incluir elementos y accesorios (flores, piedras decorativas y pintura facial).

Debido a las condiciones climáticas, los asistentes conciben una mezcla de estilos al incluir prendas como abrigos, chaquetas, capas y botas, conjuntamente con sombreros y jeans; todos estos orientados a elementos de moda y prendas de marca, al ser este último considerado como un factor relevante a la hora de planear el outfit.

De la misma manera, se considera un evento exclusivo, por lo que algunos buscan ser distinguidos o generar reputación, empleando distintos mecanismos como, por ejemplo, tener entrada a zona VIP o el uso de transporte que puede llegar a concebir clase y realce como las van e incluso helicópteros. Por consiguiente, el festival se relaciona a un evento que muchos desean vivir, llegando al punto de forjarse en algo aspiracional.

En lo que respecta a la aceptación social, esta se identifica en conexión al outfit, que no solo se emplea para llamar la atención y generar distinción, sino para ser aceptado; ocasionando la búsqueda de tendencias de festivales internacionales, comparar outfit entre asistentes e incluso despertando el deseo de verse como otros. En el mismo orden de ideas, este dilucida la forma de poder pertenecer a un grupo, al usar elementos distintivos que enuncian ser parte de un conjunto más reducido de individuos.

Como medio de exhibición, agregado al que se desarrolla durante el festival, las fotografías y videos se convierten en la forma de exponer tanto el outfit como la experiencia, siendo las redes sociales digitales el lugar para postear, lo que plantea que estas se consideran el principal conducto externo de ostentación.

\section{Discusión}

El festival logra representar una gran experiencia a pesar de la fuerte presencia de comercio y comunicación de marcas, lo que coincide con el estudio de Skandalis, Byrom \& Banister (2019), al mismo tiempo simboliza la convergencia de grandes artistas y estilos musicales, disfrute, fiesta, exhibición y libertad al forjar sentimiento de independencia de las normas sociales cotidianas (Kim \& Jamal, 2007).

De la misma forma, se encuentran aspectos hedónicos que aportan a la experiencia multisensorial, como lo sugiere (Ortegón-Cortázar y Gómez-Rodríguez, 2016) corresponde a la actividad de exploración, identificación y clasificación de las propiedades sensoriales placenteras y favorables desde la óptica del consumidor; como la selección de artistas del cartel, la organización, decoración, colores, iluminación, calidad del sonido y zonas verdes que logran incrementar el goce, disfrute y la estimulación de varios de los sentidos (vista, oído y tacto), mediante los cuales se intenta llegar a los asistentes, y de esta forma conseguir instaurar relaciones de largo plazo con el festival (Gómez-Ramírez, Manzi-Puertas y Galindo-Becerra, 2014; Hultén, 2011; Gobé, 2001).

No obstante, se descubren elementos negativos como las extensas filas, la poca suma de espacios de descanso, la congestión y dificultad de transporte. Con lo anterior, se puede decir que, comparado con algunas situaciones de compra, la participación en el festival puede llegar a provocar consecuencias hedónicas, más que efectos utilitarios tal como lo indica Laplante \& Downie (2011).

Por otra parte, la exhibición ostentosa se denota al asociar el festival como un espacio en el que se considera trascendente mostrarse y destacarse. En ese mismo orden de ideas, verse bien se concibe significativo al ser considerado un evento exclusivo donde se ve gente bonita, siendo relevante dejar una buena imagen; para ello, se accede a diferentes mecanismos para suscitar reputación con el fin de ser reconocido por el otro, proyectar clase, despertar envidia, y sentirse satisfecho de sí mismo (Lipovetsky y Roux, 2014). 
Tal como lo expresa Botton (2004), los individuos están en una constante lucha por resaltar y conseguir las mismas condiciones de otros, principalmente de sus grupos de referencia; en ese sentido, el outfit se emplea además de llamar la atención y generar distinción, para llegar a ser considerado parte de un grupo, por ello, los amigos toman gran relevancia a la hora de elegir el vestuario.

Con todo lo descrito, el evento se convierte en un encuentro tanto de diversión como de relación social, por ende, para algunas personas se hace necesario dejar una excelente imagen y conseguir ser aceptado, siendo el festival un mecanismo para lograr dichos propósitos (Veblen, 2000).

\subsection{Limitaciones y recomendaciones}

Como el estudio se desarrolló a partir de un festival pago bogotano y en la revisión de literatura no se encontró otros estudios en Colombia y Latinoamérica abordando temas de consumo hedónico y exhibición ostentosa en este tipo de eventos, se considera importante desarrollar nuevas investigaciones con metodologías cualitativas que se desplieguen desde varios contextos con festivales de la región y del mundo, implementando diseños con triangulación múltiple, que como se evidencia en esta investigación, permite mostrar los fenómenos estudiados desde distintos puntos, ofreciendo mayor validez de la información (Arias-Alpizar, 2011). Además, se hace interesante generar estudios de marketing enfocados al desarrollo de actividades Below The Line-BTL con experiencias multisensoriales que permitan generar mayor posicionamiento de las marcas patrocinadoras de este tipo de eventos.

\section{Referencias}

Arias-Alpizar, L. M. (2011). Interdisciplinariedad y Triangulación en Ciencias Sociales.

Diálogos Revista Electrónica, 10(1), 117-136.

Arráez, M., Calles, J., y Moreno de Tovar, L., (2006). La Hermenéutica: una actividad interpretativa. Sapiens. Revista Universitaria de Investigación, 7 (2), 171-18.

Bautista, N., (2011). Proceso de la investigación cualitativa epistemológica, metodología y aplicaciones. Colombia. Manual moderno. ISBN: 978-958-9446-40-9.

Botton, A., (2004). Ansiedad por el estatus [video]. YouTube. https://bit.ly/35vSggF

Choi, J., Li, Y.J., Rangan, P., Chatterjee, P. \& Singh, S.N., (2014). The odd-ending price justification effect: the influence of price-endings on hedonic and utilitarian consumption. Original empirical research. 42, 545-557. Doi: 10.1007/s11747-014-0369-6

Corbetta, P. (2003). Metodología y técnicas de investigación social. Madrid. McGraw Hill

Cultura Argentina (2017). Claves y herramientas para descifrar el ecosistema actual de la música Guia REC. Recuperado a partir de: https://www.cultura.gob.ar/guiarec_5097/

De la Espriella, R., \& Gómez-Restrepo, C., (2020). Grounded theory. Revista Colombiana de Psiquiatría (English ed.). DOI: 10.1016/j.rcpeng.2018.08.001

Del Moral, G., y Suárez-Relinque, C., (2018). La categorización familiar como técnica de apoyo al proceso de análisis que sigue la teoría fundamentada. Doi:10.1016/j.gaceta.2018.09.007

García-Ruiz, P. (2010). Consumo e identidad: un enfoque relacional. Anuario Filosófico 41 (2), 299324

Gobé, M. (2001). Emotional branding: The new paradigm for connecting brands to people. USA: Allworth Press.

Gómez-Ramírez, C., Manzi-Puertas, M. A. y Galindo-Becerra, T. (2014). Scent Marketing: A Bibliographic Review. Pensamiento \& Gestión. 37 (1), 214-254. Recuperado de: t.ly/rYA8

Gonzalo, P. (2005). El turismo cultural de Festivales y eventos como herramienta para la segmentación de la demanda turística. Investigación y Marketing. 87, 25-30. 
Heaton, J., (2004). Reworking qualitative data. SAGE Publications. London. Recuperado de: t.ly/3TE0

Hernández, R., Fernández, C y Baptista, P. (2014). Metodología de la investigación. México: Mc Graw Hill Education.

Higgins, E. T. (1997). Beyond pleasure and pain. American Psychologist, 52(12), 1280-1300. Doi: 10.1037/0003-066X.52.12.1280

Hirschman, E. \& Holbrook, M., (1982). Hedonic Consumption: Emerging Concepts, Methods and Propositions. Doi: 10.2307/1251707

Hirschman, E. (1983). Predictors of Self-Projection, Fantasy Fulfillment, and Escapism. The Journal of Social Psychology. Doi: 10.1080/00224545.1983.9712011

Hirschman, E. (1984). Experience Seeking: A Subjectivist Perspective of Consumption. Journal of Business Research. 12, 115-136.

Holbrook, M. \& Hirschman, E., (1982). The Experiential Aspects of Consumption: Consumer Fantasies, Feelings, and Fun. Doi:10.1086/208906

Holbrook, M. (2006). Consumption experience, customer value and subjective personal introspection: An illustrative photographic essay. Journal of Business Research, 59, 714-725.

Hultén, B. (2011). Sensory Marketing: The Multi-Sensory Brand-Experience Concept. European Business Review. 23 (3), 256-273. Recuperado de: t.ly/9uhX

Instituto de Políticas de Desarrollo (2013). Diez festivales en Colombia: valores e impacto. Editorial Pontificia Universidad Javeriana: Ministerio de Cultura. Recuperado de: https://bit.ly/2zZOnVz

Jaramillo, J., (2015). El boom de los conciertos musicales en Colombia: razones y limitantes. Revista Nova et Vetera. 1.5. Recuperado de: https://bit.ly/3dhTIGa

Kim, H., \& Jamal, T. (2007). Touristic quest for existential authenticity. Annals of Tourism Research, 34(1), 181-201. Doi: 10.1016/j.annals.2006.07.009

Kronrod, A., Gristein, A., \& Wathieu, L., (2011). Enjoy! Hedonic Consumption and Compliance with Assertive Messages. Journal of consumer research. 39, 51-59. Doi:10.1086/661933

Kumar, A. \& Kakar, S., (2017). Why do users prefer the hedonic but choose the Utilitarian? Investigating user dilemma of hedonic-utilitarian choice. Doi: 10.1016/j.ijhcs.2017.07.003

Laplante, A. \& Downie, J.S. (2011). The utilitarian and hedonic outcomes of music informationseeking in everyday life. Doi: 10.1016/j.lisr.2010.11.002

Larsen, G., Lawson, R., \& Todd, S., (2009). The consumption of music as self-representation in social interaction. Doi.org/10.1016/j.ausmj.2009.01.006

Lee, J., \& Shrum, L. (2012). Conspicuous Consumption versus Charitable Behavior in Response to Social Exclusion: A Differential Needs Explanation. Journal of Consumer Research, 39, 530544. Doi: $10.1086 / 664039$

Lipovetsky, G. y Roux, E., (2014). El lujo eterno (pp. 46-97). Barcelona, España: Anagrama.

Ministerio de Salud. (1993). RESOLUCION NUMERO 8430 DE 1993. Recuperado de: https://www.minsalud.gov.co/sites/rid/Lists/BibliotecaDigital/RIDE/DE/DIJ/RESOLUCION8430-DE-1993.PDF

Neme, S. y Rodríguez-González, L. (2013). Consumo simbólico: una perspectiva sociocultural en la comprensión del comportamiento del consumidor. Revista iberoamericana de Psicología: Ciencia y Tecnología, 6 (2), 27-33.

Ortegón-Cortázar, L. y Gómez-Rodríguez, A. (2016). Gestión del marketing sensorial sobre la experiencia del consumidor. Revista de Ciencias Sociales, 22 (3), 67-83. ISSN: 1315-9518. Recuperado de: t.ly/Qx0O

Perez-Truglia, R. (2013). A test of the conspicuous-consumption model using subjective well-being data. Doi: 10.1016/j.socec.2013.05.012

Strauss, A. y Corbin, J. (2002). Bases de la investigación cualitativa. Técnicas y procedimientos para desarrollar la teoría fundamentada. Colombia. Colección Contus. ISBN: 958-655-624-7. 
Prat-Forga, J., (2014). Las relaciones sociales y las motivaciones para asistir al festival de música de Peralada. Boletín de la Asociación de Geógrafos Españoles. 66, 207-221. ISSN: 0212 9426. Recuperado de: bre.is/5jd2czXS

Quintana, A. y Montgomery, W. (2006). Metodología de Investigación Científica Cualitativa. Psicología: Tópicos de actualidad. Recuperado de: shorturl.at/estIT

Semana (2014). Estéreo Picnic: de la quiebra al éxito. Recuperado de https://bit.ly/3b1oXDH

Skandalis, A., Byrom, J. \& Banister, E. (2019). Experiential marketing and the changing nature of extraordinary experiences in post-postmodern consumer culture. Journal of Business Research. 97, 43-50. Doi: 10.1016/j.jbusres.2018.12.056

Tabuenca, M., (2002). ler Congreso Internacional de Turismo Cultural. Recuperado de: http: //www.turismo-cultural.org.

Taylor, S. y Bogdan R., (2000) Introducción a los métodos cualitativos de investigación. Tercera edición. Paidós básica. Recuperado de: shorturl.at/oBDF2

Tikitakas (2019). Lo bueno y lo malo que dejó el festival. Recuperado a partir de: https://bit.ly/3aXV5bq

Veblen, T. (2000). Teoría de la clase ociosa. Canadá. Ediciones elaleph.com. Recuperado de: https://bit.ly/35yAdX

Walker-Janzen, W., (2016). Algunas consideraciones para el uso de la metodología cualitativa en investigación social. Foro educacional, 27, 13-32. 